\title{
Lyssaviruses in Insectivorous Bats, South Africa, 2003-2018
}

\author{
Jessica Coertse, Colyn S. Grobler, Claude T. Sabeta, Ernest C.J. Seamark, \\ Teresa Kearney, Janusz T. Paweska, Wanda Markotter
}

We detected 3 lyssaviruses in insectivorous bats sampled in South Africa during 2003-2018. We used phylogenetic analysis to identify Duvenhage lyssavirus and a potentially new lyssavirus, provisionally named Matlo bat lyssavirus, that is related to West Caucasian bat virus. These new detections highlight that much about lyssaviruses remains unknown.

L yssaviruses cause fatal encephalitic disease in umammals; 6 viral species have been implicated in human deaths (1). Bats are the primary hosts for members of the Lyssavirus genus, which belongs to the family Rhabdoviridae. Researchers have described 17 lyssavirus species, and a putative species is awaiting formal classification (1). The genus can be divided into $\geq 3$ phylogroups on the basis of genetic, immunogenic, and pathogenic properties (2). Rabies vaccines and postexposure prophylaxis protect against infection by members of phylogroup I but provide limited protection against phylogroups II or III (3).

In Africa, 6 lyssaviruses are in circulation: rabies virus, which is associated with terrestrial carnivores; Duvenhage virus (DUVV), which is associated with insectivorous bats, specifically the Egyptian slit-faced bat (Nycteris thebaica); Lagos bat virus, which is associated with various species of frugivorous bats; Mokola virus, for which the reservoir host is unknown; Shimoni bat virus, which is associated with the striped leaf-nosed bat (Macronycteris vittatus); and Ikoma lyssavirus, for which the reservoir host is unknown (1). Only rabies virus, DUVV, and Mokola virus have

Author affiliations: University of Pretoria, Pretoria, South Africa (J. Coertse, C.S. Grobler, T. Kearney, J.T. Paweska,

W. Markotter); National Institute of Communicable Diseases of the National Health Laboratory Service, Sandringham, South Africa (J. Coertse, J.T. Paweska); Onderstepoort Veterinary Institute, Pretoria (C.T. Sabeta); AfricanBats NPC, Pretoria (E.C.J. Seamark, T. Kearney); Ditsong National Museum of Natural History, Pretoria (T. Kearney)

DOI: https://doi.org/10.3201/eid2612.203592 been associated with human deaths on the continent. Lyssavirus surveillance in Africa is inadequate. As a result, genetic diversity, geographic distribution, and host species associations of lyssaviruses are poorly understood (1). However, this information is crucial for making treatment decisions, especially in resource-limited settings (1).

We report the results of 16 years of surveillance of insectivorous bats in South Africa. We used genetic characterization to identify DUVV and a potential novel lyssavirus from phylogroup III.

\section{The Study}

During 2003-2018, we tested 605 insectivorous bats (Appendix Tables 1, 2, https:/ / wwwnc.cdc.gov/EID/ article/26/12/20-3592-App1.pdf) of 41 species across South Africa (Figure 1; Appendix Tables 1, 2). Most bats were collected as part of a broader biosurveillance program in collaboration with bat taxonomists for species identification and classification. Among the bats collected, 562 appeared healthy and 28 were dead. Another 12 exhibited signs of disease or abnormal behavior, and 3 had been involved in human contact; we submitted these samples for rabies testing.

We anesthetized the sampled bats with isoflurane inhalant (Safeline Pharmaceuticals, https://safeline. co.za), exsanguinated them by cardiac puncture, and then performed full necropsies. We identified bats on the basis of morphologic (4) and genetic characteristics (5). The sampling protocol was approved by the University of Pretoria Animal Ethics Committee (approval no. EC054-14). Permission to conduct research was obtained from the Department of Agriculture, Land Reform and Rural Development (formerly Department of Agriculture, Forestry, and Fisheries) of the Republic of South Africa under Section 20 of the Animal Diseases Act 1984 with additional provincial permits granted (Appendix Table 3).

We extracted total RNA from the bats' brain material and subjected it to real-time reverse transcription PCR selective for $126 \mathrm{bp}$ of the nucleoprotein gene 


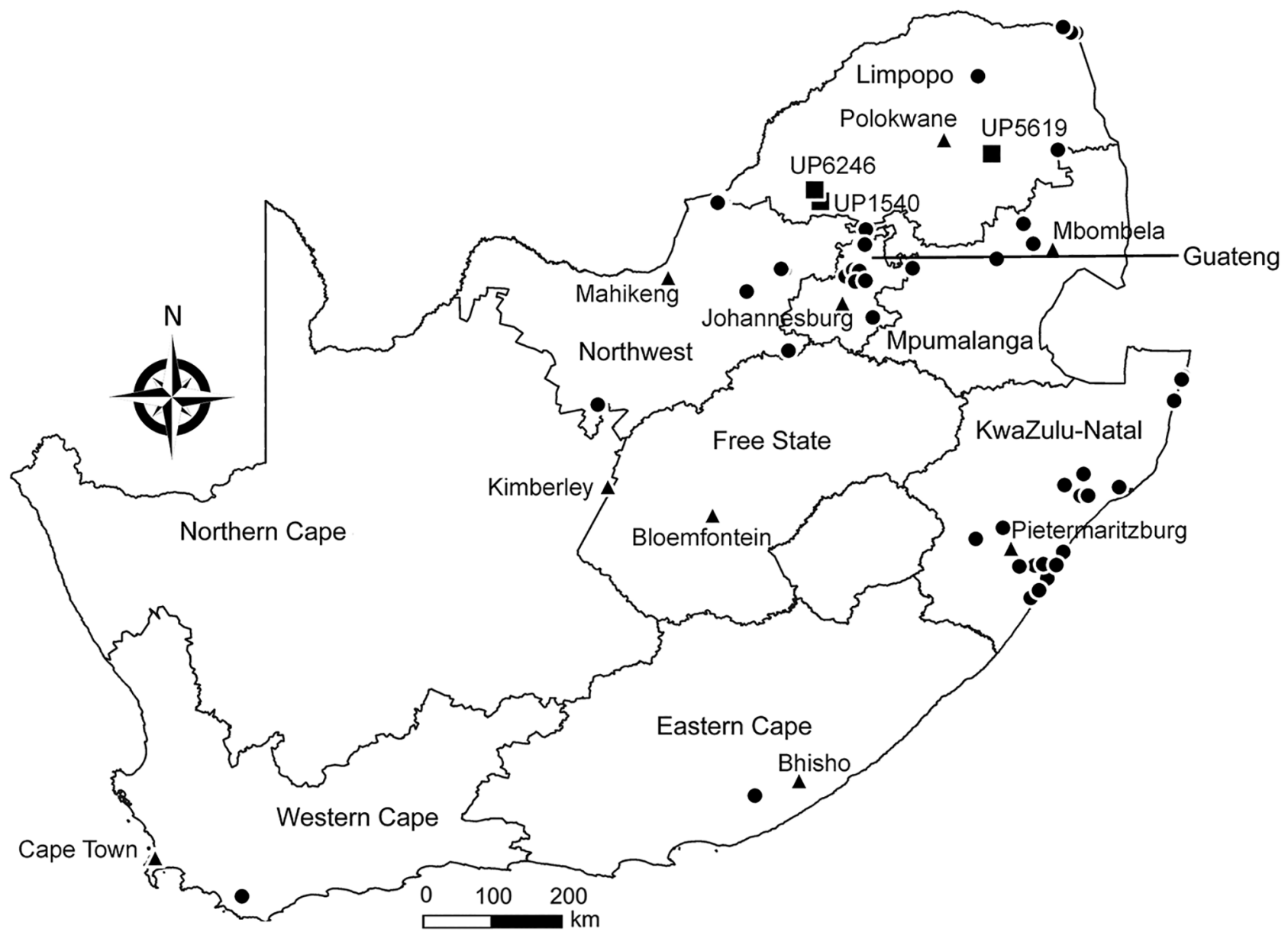

Figure 1. Locations of bat collection sites, South Africa, 2003-2018. Circles indicate collection sites, squares indicate sites with lyssavirus-positive bats, and triangles indicate capitals.

(6). Samples from 3 bats tested positive for lyssavirus RNA; we sequenced the amplicons (7). One of these samples came from an Egyptian slit-faced bat (sample no. UP1540) collected in 2012 from the Rooiberg area, Limpopo. This bat, which was collected by members of a bat interest group in August 2012, tested positive for DUVV (Figure 1). We also obtained sequences of a potentially novel lyssavirus in samples from 2 apparently healthy Natal long-fingered bats (Miniopterus natalensis, sample nos. UP5619 and UP6246); these sequences were distantly related to West Caucasian bat virus (WCBV). We collected these samples from Matlapitsi cave, Limpopo, in 2015 and Madimatle cave, Limpopo, in 2016 (Figure 1).

To infer the phylogeny, we used the complete nucleoprotein gene sequences of the 3 lyssaviruses according to a method described previously (8). We used Bayesian inference to compare our samples with representative sequences from GenBank (Appendix Table 4). We analyzed the dataset with BEAST (9); we used the general time-reversible substitution model as determined by jmodeltest2 (10), invariant sites, and gamma distribution. We assumed an underlying coalescent process with constant population size and Markov chain Monte Carlo chains of 50 million generations.

The results indicated that the sequences from Natal long-fingered bats were most closely related to, but distinct from, WCBV (Figure 2); they might belong to a novel species of Lyssavirus, provisionally called Matlo bat lyssavirus (MBLV). These 2 novel sequences had a shared nucleotide identity of $99.2 \%$. Compared with other lyssaviruses, they shared the highest nucleotide identity with WCBV $(80.9 \%-81 \%)$ and the lowest with Ikoma lyssavirus (70.5\%). The DUVV sequence was $91.6 \%$-99.3\%, similar to previously described DUVV sequences (Table).

\section{Conclusions}

From 2003-2018, we detected 3 lyssavirus infections in insectivorous bats from South Africa, indicating 
active but low-level circulation of lyssaviruses in this population. The Egyptian slit-faced bat is the only species of bat that has been conclusively linked with DUVV (11). Our finding is 1 of only 6 known DUVV cases, 3 of which caused fatal infection in humans (1). This finding suggests that these infections are underreported. The Egyptian slit-faced bat is widely distributed in Africa. It co-roosts with bats of various other species and switches roosts frequently, implicating a wider potential to infect other species (12).

We detected a novel lyssavirus, MBLV, that belongs to phylogroup III and is most closely related to a sequence of WCBV that was isolated from a common long-fingered bat (Miniopterus schreibersii) from the Russian Caucasus in 2002 (13). The nucleotide identity for MBLV falls within the species demarcation criteria determined by the International Committee for the
Taxonomy of Viruses of $80 \%-82 \%$ for the complete nucleoprotein gene (2). If MBLV is not pronounced a new lyssavirus species, this virus would be a distinct lineage of WCBV. In 2006-2007, detection of virus neutralizing antibodies (seroprevalence 17\%-26\%) against WCBV in Miniopterus bats in Kenya spurred speculation that WCBV, or a closely related virus, was circulating in Africa (14). The bat family Miniopteridae, which includes $\geq 23$ species in Africa, does not host known lyssaviruses in phylogroup I or II (12). The long-fingered bats are widely distributed throughout Africa (12).

Models have demonstrated that WCBV can cause fatal encephalitis in animals; commercial human and veterinary vaccines do not offer significant protection (3). Considering genetic diversity and phylogenetic grouping, we speculate that current vaccines will probably provide little to no protection against

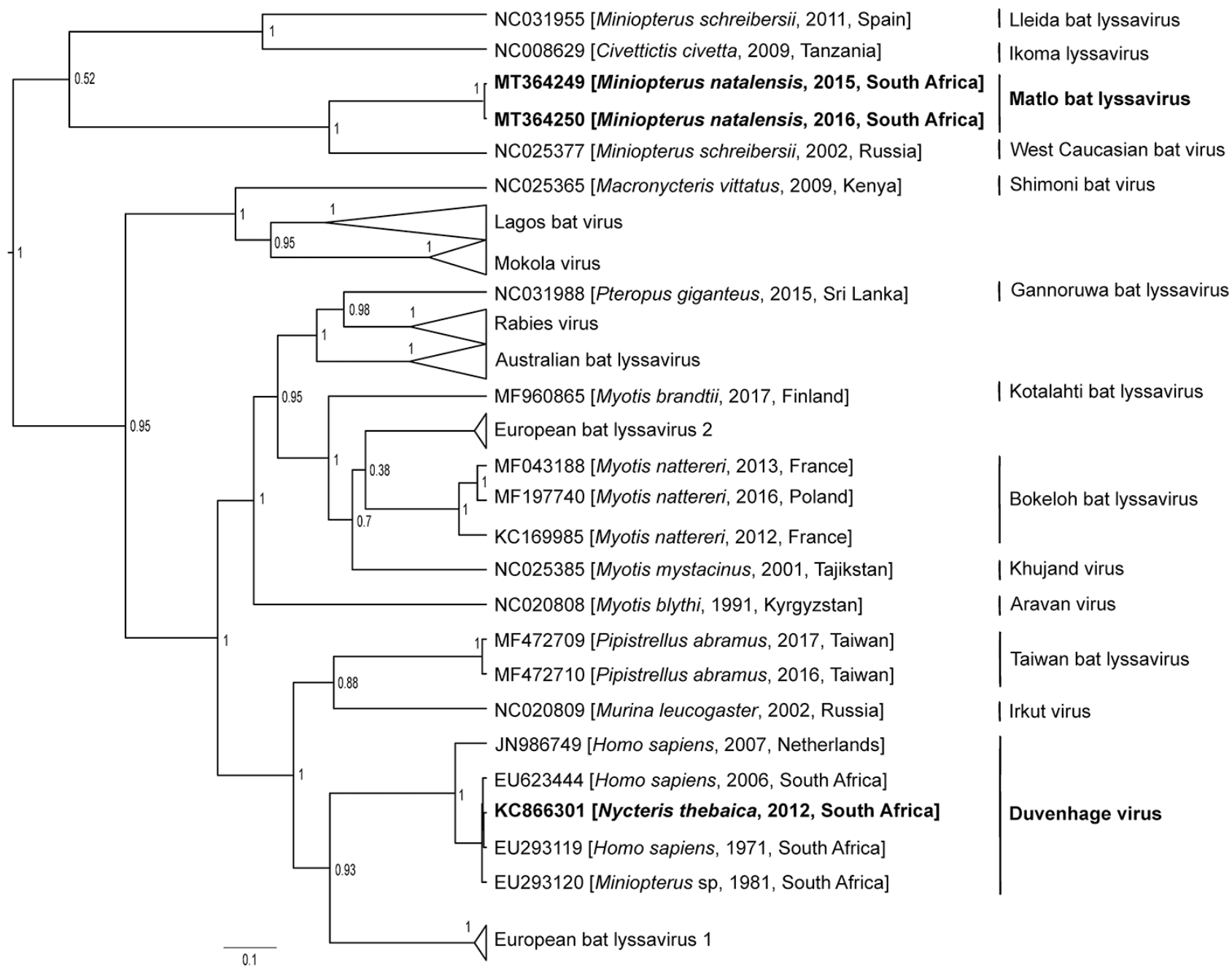

Figure 2. Phylogenetic reconstruction by Bayesian inference of nucleoprotein gene sequences of lyssavirus sequences from bats collected in South Africa, 2003-2018 (bold), and other representative lyssaviruses. Node numbers indicate posterior probabilities. GenBank accession number, host species, year of detection, and country of origin are indicated for each sequence. Scale bar indicates number of substitutions per site. 
Lyssaviruses in Bats, South Africa, 2003-2018

Table. Genetic similarities of 3 lyssaviruses found in insectivorous bats in South Africa, 2003-2018, and representative lyssavirus species from GenBank*

\begin{tabular}{|c|c|c|c|c|c|c|}
\hline \multirow[b]{3}{*}{ Virus } & \multicolumn{6}{|c|}{$\%$ Similarity } \\
\hline & \multicolumn{2}{|c|}{ UP5619 (MT364249) } & \multicolumn{2}{|c|}{ UP6246 (MT364250) } & \multicolumn{2}{|c|}{ UP1540 (KC866301) } \\
\hline & Nucleotide & Amino acid & Nucleotide & Amino acid & Nucleotide & Amino acid \\
\hline Aravan virus & 72.7 & 83.3 & 72.6 & 84.0 & NA & NA \\
\hline Australian bat lyssavirus & $72.7-74.4$ & $82-83.3$ & $72.8-74.7$ & $82.4-83.7$ & NA & NA \\
\hline Bokeloh bat lyssavirus & $72.9-73.3$ & 81.5 & $73-73.4$ & 81.8 & NA & NA \\
\hline European bat lyssavirus 1 & $71.1-72.4$ & $82.2-82.4$ & $71.2-72.5$ & $82.7-82.9$ & NA & NA \\
\hline European bat lyssavirus 2 & $71-72.4$ & $80.0-80.7$ & $71-72.4$ & $80.2-80.9$ & NA & NA \\
\hline Gannoruwa bat lyssavirus & 70.6 & 83.1 & 70.6 & 83.5 & NA & NA \\
\hline Ikoma lyssavirus & 70.5 & 79.5 & 70.5 & 79.5 & NA & NA \\
\hline Irkut virus & 72 & 83.3 & 72.4 & 83.8 & NA & NA \\
\hline Khujand virus & 72.5 & 81.3 & 72.5 & 81.8 & NA & NA \\
\hline Kotalahti bat lyssavirus & 71.9 & 81.8 & 71.9 & 82.4 & NA & NA \\
\hline Lagos bat virus & $71.1-74.2$ & $82.6-84$ & $71.3-74.5$ & $83.1-84.4$ & NA & NA \\
\hline Lleida bat lyssavirus & 71.3 & 81.5 & 71.4 & 81.5 & NA & NA \\
\hline Mokola virus & $72.2-73.8$ & $82.4-83.7$ & $72.3-73.7$ & $82.8-84.2$ & NA & NA \\
\hline Rabies virus & $71.1-72.4$ & $80.6-82$ & $71-72.2$ & $80.8-82.4$ & NA & NA \\
\hline Shimoni bat virus & 73.1 & 83.5 & 73.6 & 84.2 & NA & NA \\
\hline Taiwan bat lyssavirus & $73.6-73.9$ & 83.3 & $73.7-73.9$ & 83.8 & NA & NA \\
\hline West Caucasian bat virus & 80.9 & 95.5 & 81 & 96.2 & NA & NA \\
\hline \multicolumn{7}{|l|}{ DUVVs } \\
\hline EU293119 & 72.9 & 84 & 72.9 & 84.4 & 99.3 & 99.5 \\
\hline EU293120 & 72.9 & 84 & 72.9 & 84.4 & 98.5 & 99.5 \\
\hline JN986749 & 73 & 84 & 73 & 84.4 & 91.6 & 99.3 \\
\hline EU623444 & 72.8 & 84 & 72.8 & 84.4 & 98.6 & 99.3 \\
\hline
\end{tabular}

infection with MBLV (as with WCBV). Because of the lack of diagnostic capability in Africa (6), the potential threat of this virus is unknown. Continued surveillance and development of improved pharmaceuticals are necessary for the prevention of these infections. We observed a low prevalence $(0.5 \%)$, similar to other lyssaviruses (1). Additional longitudinal surveillance, including serologic testing, among bats of this species and other potential hosts must be implemented to determine if Natal long-fingered bats are the reservoir host of MBLV. This study did not obtain equally representative samples of all bat species from all sampling sites; MBLV might exist in other bat species in South Africa.

In summary, the mechanisms of lyssavirus maintenance in bats is still unknown and could be influenced by various environmental and ecologic factors (1). Additional surveillance and comparative seroprevalence studies are needed to establish the host range and distribution of MBLV and other lyssaviruses. Although the public health impact of MBLV is currently unknown, DUVV can cause fatal infection and should be taken seriously. Surveillance is needed to understand the epidemiology and diversity of bat lyssaviruses and inform prevention efforts.

\section{Acknowledgments}

We thank the students and staff of the Centre for Viral Zoonoses, University of Pretoria, for technical assistance during fieldwork. We also thank the bat rehabilitator community, the bat interest groups for submission of bats for testing, and the Ga Mafefe community in the Limpopo Province for supporting our research at Matlapitsi cave. All participants in this project were vaccinated against rabies according to the World Health Organization preexposure prophylaxis guidelines. We also thank Motjoli Resources, Aquila Steel, and Aquila Resources for their support and permission to conduct surveillance at Meletse.

This research is supported in part by the South African Research Chair Initiative (held by Wanda Markotter) of the Department of Science and Innovation and administered by the National Research Foundation of South Africa (UID: 98339). The National Research Foundation funded the equipment based at the DNA Sanger sequencing facility in the Faculty of Natural and Agricultural Sciences, University of Pretoria (UID: 78566). This research was also supported in part by Cooperative Agreement no. 5 NU2GGH001874-02-00, funded by the US Centers for Disease Control and Prevention. These contents are solely the responsibility of the authors. C.S.G. was additionally supported by the Poliomyelitis Research Foundation (grant no. 18/54).

\section{About the Author}

Dr. Coertse is a postdoctoral fellow in the Centre for Viral Zoonoses at the University of Pretoria. Her research interests are focused on zoonoses, particularly those related to lyssaviruses and bats. 


\section{References}

1. Markotter W, Coertse J. Bat lyssaviruses. Rev Sci Tech. 2018;37:385-400. https:/ / doi.org/10.20506/rst.37.2.2809

2. Walker PJ, Blasdell KR, Calisher $\mathrm{CH}$, Dietzgen RG, Kondo H, Kurath G, et al.; ICTV Report Consortium. ICTV virus taxonomy profile: Rhabdoviridae. J Gen Virol. 2018;99:447-8. https://doi.org/10.1099/jgv.0.001020

3. Hanlon CA, Kuzmin IV, Blanton JD, Weldon WC, Manangan JS, Rupprecht CE. Efficacy of rabies biologics against new lyssaviruses from Eurasia. Virus Res. 2005; 111:44-54. https:// doi.org/10.1016/j.virusres.2005.03.009

4. Meester J. Classification of southern African mammals. Pretoria (South Africa): Transvaal Museum Monograph; 1986. p. 1-359.

5. Folmer O, Black M, Hoeh W, Lutz R, Vrijenhoek R. DNA primers for amplification of mitochondrial cytochrome $\mathrm{C}$ oxidase subunit I from diverse metazoan invertebrates. Mol Mar Biol Biotechnol. 1994;3:294-9.

6. Coertse J, Weyer J, Nel LH, Markotter W. Reverse transcription recombinase polymerase amplification assay for rapid detection of canine associated rabies virus in Africa. PLoS One. 2019;14:e0219292. https:/ / doi.org/10.1371/ journal.pone.0219292

7. Coertse J, Weyer J, Nel LH, Markotter W. Improved PCR methods for detection of African rabies and rabies-related lyssaviruses. J Clin Microbiol. 2010;48:3949-55. https://doi.org/10.1128/JCM.01256-10

8. Markotter W, Kuzmin I, Rupprecht CE, Randles J, Sabeta CT, Wandeler AI, et al. Isolation of Lagos bat virus from water mongoose. Emerg Infect Dis. 2006;12:1913-8. https://doi.org/10.3201/eid1212.060514

9. Drummond AJ, Suchard MA, Xie D, Rambaut A. Bayesian phylogenetics with BEAUti and the BEAST 1.7. Mol Biol Evol. 2012;29:1969-73. https://doi.org/10.1093/molbev/mss075

10. Darriba D, Taboada GL, Doallo R, Posada D. jModelTest 2: more models, new heuristics and parallel computing. Nat Methods. 2012;9:772-772. https://doi.org/10.1038/nmeth.2109

11. Foggin C. Rabies and rabies-related viruses in Zimbabwe: Historical, virological and ecological aspects. Harare (Zimbabwe): University of Zimbabwe; 1988. p. 186-221.

12. AfricanBats NPC. African Chiroptera report 2019. 2019 [cited 2020 Apr 13]. https:/ / africanbats.org/publication/ african-chiroptera-report-2019

13. Botvinkin AD, Poleschuk EM, Kuzmin IV, Borisova TI, Gazaryan SV, Yager P, et al. Novel lyssaviruses isolated from bats in Russia. Emerg Infect Dis. 2003;9:1623-5. https://doi.org/10.3201/eid0912.030374

14. Kuzmin IV, Niezgoda M, Franka R, Agwanda B, Markotter W, Beagley JC, et al. Possible emergence of West Caucasian bat virus in Africa. Emerg Infect Dis. 2008;14:1887-9. https://doi.org/10.3201/eid1412.080750

Address for correspondence: Wanda Markotter, Centre for Viral Zoonoses, Department of Medical Virology, Faculty of Health Sciences, Private Bag X323, Room 2-66, Pathology Bldg, Prinshof Campus, University of Pretoria, Gezina, Pretoria 0031, South Africa; email: wanda.markotter@up.ac.za

\section{EID Podcast Plague in a Dog}

Some might think the plague is a relic of the Middle Ages. But Yersinia pestis still lingers, and has even infected man's best friend.

m.t. has ev

In this EID podcast, Dr. Joshua Daniels, a bacteriologist at Colorado State University's Veterinary Diagnostic Laboratory, explains how doctors diagnosed this unusual infection.

\section{Visit our website to listen: EMERCING}

https://tools.cdc.gov/medialibrary/index.aspx\#/media/id/398724 INFECTIOUS DISEASES 